\section{Fracción de óxido nítrico exhalado: una herramienta clínica para las enfermedades pulmonares}

\author{
LUZ MIRANDA $^{1, \mathrm{a}}$, JULIA GUERRERO ${ }^{1,2,3, \mathrm{~b}}$
}

\section{Measurement of exhaled nitric oxide fraction in lung diseases}

Exhaled Nitric Oxide fraction measurement is a new method for the evaluation of respiratory diseases. It has good correlation with airway inflammation and decreases with the administration of corticosteroids. It is useful as a complement for the diagnosis of asthma, Chronic Obstructive Pulmonary Disease, Cystic Fibrosis and Primary Ciliary Dyskinesia among other respiratory diseases that generate inflammation in the airway. Its assessment is easy, non-invasive, and safe, and the result is obtained immediately. It can be used routinely to evaluate the response and adherence to treatments. This article reviews the biology of Nitric Oxide, and the measurement, interpretation, and main clinical uses of Exhaled Nitric Oxide Fraction.

(Rev Med Chile 2021; 149: 1173-1181)

Key words: Biomarkers; Nitric Oxide; Respiratory Tract Diseases.
'Laboratorio de Inmunomodulación

Neuroendocrina del Programa

Disciplinario de Fisiología y Biofísica-ICBM

Facultad de Medicina, Universidad de

Chile. Santiago, Chile.

${ }^{2}$ Servicio de Paciente Crítico y

Departamento de Medicina Interna-

Hospital Clínico, Universidad de Chile.

Santiago, Chile.

${ }^{3} \mathrm{UCl}$ Adultos Clínica Alemana de

Santiago, Chile.

aKinesióloga y alumna de Programa

Magíster Fisiología de la Escuela

Postgrado, Facultad Medicina,

Universidad de Chile.

bPhD.

Trabajo no recibió financiamiento.

Recibido el 3 de enero de 2020, aceptado el 11 de junio de 2021.

Correspondencia a:

Dra. Julia Guerrero

Programa Disciplinario de Fisiología y

Biofísica.

Facultad Medicina - Universidad de Chile.

Avenida Independencia 1027-

Independencia.

jguerrer@uchile.c
$\mathrm{E}$ n las enfermedades respiratorias, el óxido nítrico (NO) es considerado un biomarcador de inflamación ${ }^{1}$. Estudios han confirmado que respecto de controles sanos, la fracción de óxido nítrico exhalado (FeNO) se encuentra elevado en pacientes asmáticos ${ }^{2,3}$. Además, los valores de FeNO se correlacionan con la presencia de inflamación en la vía aérea y disminuye con el tratamiento de glucocorticoides inhalados ${ }^{4}$ constituyendo una herramienta útil para predecir la respuesta al tratamiento . $^{5}$.

Las pruebas convencionales de función pulmonar tales como la valoración del volumen espiratorio forzado en el primer segundo $\left(\mathrm{VEF}_{1}\right)$, se asocian de modo indirecto con la inflamación de las vías respiratorias. La cuantificación de FeNO, junto con la valoración de la inflamación en la vía aérea, ofrece ventajas adicionales tales como detección del carácter eosinofílico de la inflamación de las vías respiratorias, predecir la respuesta al tratamiento con glucocorticoides y capacidad estimar la adherencia a la terapia con corticosteroides $^{6,7}$.

Los avances tecnológicos y la estandarización de la técnica han simplificado la valoración de FeNO, facilitando su uso clínico tanto en pacientes adultos como pediátricos. Dado que la cuantificación de FeNO constituye un método no invasivo, simple y seguro para valorar la inflamación de las vías respiratorias, es considerada una valiosa nueva herramienta clínica que se suma a las técnicas tradicionales para la evaluación y ajuste terapéutico de pacientes con enfermedades inflamatorias de las vías respiratorias. A continuación, realizamos una revisión del rol de $\mathrm{NO}$ en algunas patologías pulmonares y el rol de FeNO en la clínica.

\section{Biología del NO}

El NO, originalmente llamado factor relajante derivado del endotelio, es un radical libre con 
función de señalización y que está involucrado en amplia gama de funciones celulares. Su descubrimiento y la comprensión de su fisiología motivó la obtención del premio Nobel de Medicina y Fisiología a los farmacólogos norteamericanos R. Furchgott, L. Ignarro y F. Murad en 1998.

El NO es una molécula gaseosa catalizada por la enzima Óxido Nítrico Sintasa (NOS). Se reconocen tres isoformas de NOS: NOS endotelial (eNOS o NOS3), NOS neuronal (nNOS o NOS1) y NOS inducible (iNOS o NOS2). NOS1 y NOS3 son enzimas constitutivas controladas por $\mathrm{Ca}^{2+} /$ calmodulina intracelular; mientras que NOS2 es inducible a nivel de transcripción génica e independiente de $\mathrm{Ca}^{2+}$, su expresión es inducida en respuesta a mediadores (pro) inflamatorios tales como citoquinas (TNF $\alpha$, IL-1, INF $\gamma$ ), virus, bacterias, alérgenos y contaminantes ${ }^{8,9}$ (Figura 1 ). Los glucocorticoides inhiben la activación transcripcional de NOS2, efecto que no se observa en las enzimas NOS1 y NOS3 ${ }^{9,10}$.

En el sistema ventilatorio, se sabe que las NOS son expresadas en una amplia variedad de células ${ }^{11}$ : NOS1 es expresada en las fibras No Adrenérgicas- No Colinérgicas (NANC) generando NO que actúa como factor relajante del músculo liso; NOS3 ha sido identificada en el endotelio pulmonar, células del epitelio bronquial y alveolar ${ }^{12}$ relacionado su expresión con el movimiento ciliar $^{8}$ y la expresión de la forma inducible de la NOS (NOS2), ha sido identificada en las células epiteliales, endoteliales, músculo liso vascular, fibroblastos, mastocitos y neutrófilos ${ }^{13}$. En respuesta a la activación transcripcional de la NOS2, la producción de NO aumenta desde concentraciones

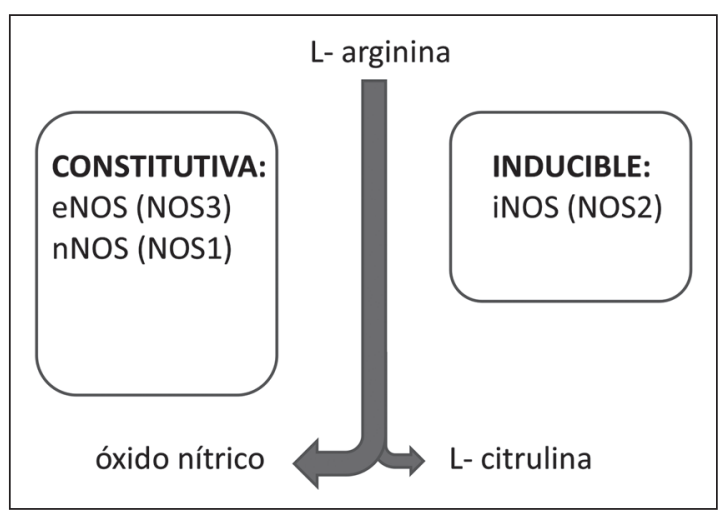

Figura 1. Óxido Nítrico Sintasa (NOS) y Biosíntesis de Óxido Nítrico (NO). nanomolares a niveles significativamente más altos a los observados en condiciones basales.

En general, las acciones biológicas de NO pueden ser clasificada como dependiente de cGMP o independiente de cGMP. La primera hace referencia al efecto de NO mediado por guanilil ciclasa soluble, en ella la unión de $\mathrm{NO}$ al Fe ${ }^{2+}$ a la enzima modifica su actividad llevando a la conversión de GTP a cGMP, que a su vez activa kinasas dependiente de cGMP. Los efectos biológicos mediados por este mecanismo incluyen relajación de músculo liso vascular y de vías aéreas. El segundo mecanismo -independiente de cGMP- requiere la participación de canales de $\mathrm{K}^{+}$dependientes de calcio $^{14,15}$.

La producción de NO puede ser inhibida por bloqueo de la NOS, por la administración de un sustrato alternativo que inhibe la producción de $\mathrm{NO}$ o por la inducción de cualquier tipo de arginasa que disminuyen la biodisponibilidad de L-arginina necesario para la producción de $\mathrm{NO}$ mediada por NOS.

\section{NO e inflamación pulmonar}

Las tres isoformas de NOS están localizadas en el sistema ventilatorio $y$, por tanto, participan en la regulación fisiológica de las vías respiratorias a través de la producción cooperativa de NO. Modificaciones en su actividad pueden conducir a cambios en el nivel de $\mathrm{NO}$ y contribuir a la patogénesis de varias enfermedades respiratorias. Pequeñas cantidades de NO producidas por formas constitutivas de NOS están involucradas en la regulación de los procesos fisiológicos, mientras que los altos niveles de NO poseen el efecto tóxico (Tabla $1)^{16,17}$. En general, los procesos biológicos pueden ser agrupados como i) dilatación o relajación de las vías respiratorias por efecto de éste en células propias de la vía aérea; ii) dilatación de células del músculo liso vascular; iii) secreción de mucus y iv) respuesta inmune, entre otros (Tabla 1 ).

El desarrollo tecnológico en la actualidad permite que la valoración de FeNO sea un método estandarizado y simple, facilitando su uso en clínica (Figura 2). Sin embargo, la variabilidad inter-individuos reportada para este biomarcador ha sido el principal limitante para su utilidad clínica.

En general, en la valoración de pruebas de función pulmonar en individuos sanos son factores 
Tabla 1. Acciones biológicas de NO en el sistema respiratorio

\begin{tabular}{|ll|}
\hline Fisiológicos & Patológicos \\
\hline Broncodilatación & Hiperreactividad bronquial \\
\hline Producción de surfactante & Vasodilatación \\
\hline Secreción de mucus & Hipersecreción de mucus \\
\hline Estimulación motilidad ciliar & Inhibición motilidad ciliar \\
\hline Neurotransmisión & Producción de radicales libres \\
\hline Efecto anti-inflamatorio & Efecto pro-inflamatorio \\
\hline
\end{tabular}

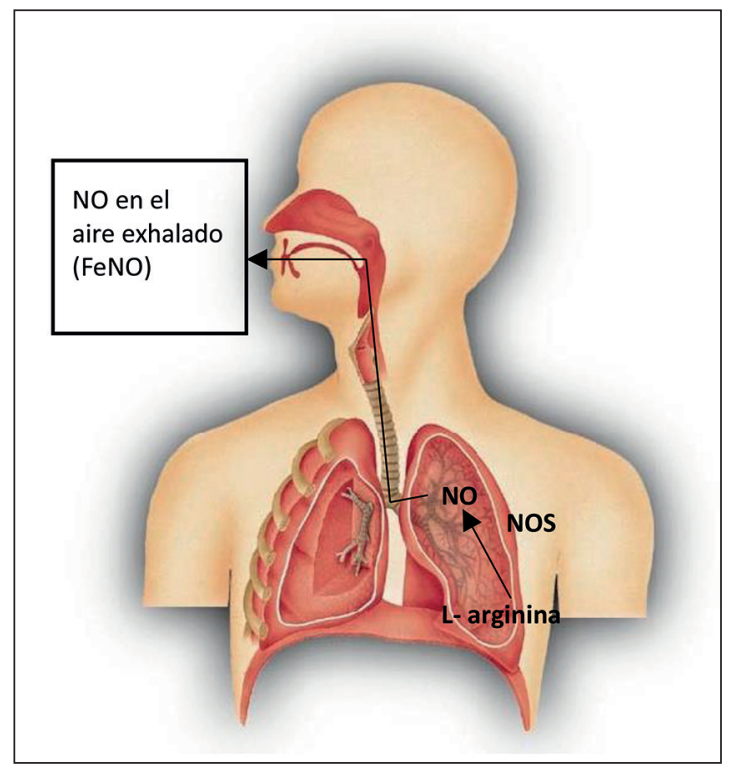

Figura 2. Óxido nítrico exhalado.

importantes la edad, tamaño corporal, factores genéticos, sexo, etnicidad, factores socioeconómicos, historia familiar de asma, atopia y exposición a contaminantes ambientales tales como el tabaco ${ }^{13,18}$. Interesantemente, en la producción de NO valorada como FeNO, se ha descrito una importante variabilidad entre sujetos lo cual parece estar relacionado con la fuente de producción de $\mathrm{NO}$ en el tejido pulmonar. Autores han propuesto que el NO exhalado estaría compartimentado entre la vía aérea y alveolos, de modo que, este último correspondería a la fuente biológica de NO indicativa de inflamación pulmonar distal ${ }^{14,19}$ $y$, por tanto, originada en respuesta a la inducción de la actividad de la NOS2 ${ }^{15,20}$.
Por otra parte, estudios in vitro demostraron que la inducción de NOS2 difiere en células epiteliales del tejido pulmonar: mientras las células de la vía central responden con inducción de NOS2 ante el estímulo de IL-13, las de la vía aérea pequeña (origen alveolar) no responden a esta citoquina, sino que desarrollan una rápida inducción de NOS2 frente a un estímulo compuesto de una mezcla de citoquinas proinflamatorias (IL- $1 \beta$, TNF- $\alpha$ e IFN- $\gamma)^{16,21}$. Estas características de la activación de NOS junto a datos obtenidos en la investigación preclínica han posicionado al FeNO como un instrumento clínicamente útil para monitorizar el curso clínico de patologías respiratorias como el asma en adultos y pacientes pediátricos y aun cuando existe una activa e interesante discusión en la literatura en relación al efectivo rol de FENO en clínica, sociedades científicas como la American Thorax Society (ATS) y European Respiratory Society (ERS) han apoyado su uso ${ }^{12,13,18,22}$.

Por otra parte, autores han planteado que los cambios en los valores de FeNO también pueden ser explicados por el cambio en el calibre de las vías respiratorias, de modo que este biomarcador -más que un biomarcador puramente inflamatorio- refleja la integración entre inflamación de las vías respiratorias y cambios en la función pulmonar ${ }^{19,23}$. Otros autores, sin bien concuerdan con los cut-points propuestos para FENO en asma, señalan que existe un importante número de pacientes asmáticos con valores de FENO intermedios y, por tanto, excluidos de las recomendaciones de la $\mathrm{ATS}^{24,25}$. Interesantemente, la revisión sistemática de Essat $\mathrm{M}$ et al. publicada en 2016 y que incluyó 6 RCT, mostró que en adultos, el tratamiento de asma guiado por FeNO consiguió una reducción 
estadísticamente significativa en las tasas de exacerbaciones/año por persona y también en mujeres cursando el segundo semestre de embarazo en quienes, además, permitió reducir el uso de corticoides inhalados ${ }^{26}$. Por otra parte, recientemente un novedoso estudio clínico propone la identificación de pacientes con enfermedad pulmonar obstructiva crónica (EPOC) con perfil obstructivo de la vía aérea y respuesta satisfactoria a la terapia broncodilatora, ampliando así el espectro de patologías respiratorias que podrían beneficiarse con la monitorización de $\mathrm{FENO}^{27}$.

\section{Cuantificación de FeNO e Interpretación de Resultados}

Para cuantificar FeNO se utilizan dispositivos de análisis en línea que por métodos como quimioluminiscencia, entregan el resultado en forma inmediata $^{28}$. Estas mediciones requieren que el sujeto realice una respiración única inhalando a capacidad pulmonar total y exhalando a flujo de $50 \mathrm{ml} / \mathrm{s}$ durante 6 segundos, tiempo requerido para la estabilización que los niveles de FeNO. La constancia del flujo exhalado se consigue a través de una resistencia en línea la que, a su vez, proporciona una retroalimentación al paciente por una interfaz gráfica en la computadora. Las maniobras se repiten al menos dos veces para asegurar la reproducibilidad ${ }^{29}$.

La variabilidad de los rangos de referencia en sujetos normales adultos y niños ha motivado que la ATS recomiende interpretar los valores deFeNO de acuerdo a puntos de corte pre establecidos ${ }^{16}$ : valores de FeNO menores a $25 \mathrm{ppb}(<20 \mathrm{ppb}$ para pediatría) indican ausencia de inflamación en la vía aérea, mientras que valores mayores a 50 ppb (o > 35 ppb para pediatría) concuerdan con presencia de proceso inflamatorio respondedor a corticosteroides. Valores entre 25 - $50 \mathrm{ppb}$ (20-35 ppb para pediatría) deben ser interpretados en forma individualizada ${ }^{16}$.

Respecto de la utilidad de FeNO para guiar la terapia con corticosteroides inhalados, la recomendación de expertos propone considerar los valores basales individuales de FENO, es decir, más que un valor aislado, se propone valorar el cambio de los valores de FENO en forma individualizada. Así, se sugiere considerar significativo cambios de FeNO $>20 \%$ cuando el valor basal es $>50 \mathrm{ppb}$ mientras que cambios $>10 \%$ serán significativos para sujetos con FeNO basal $<50 \mathrm{ppb}^{16}$.

Estos puntos de corte fueron determinados utilizando equipos estacionarios de quimioluminescencia como Sievers NOA280 ${ }^{\circledR}$, sin embargo, actualmente se encuentran disponible en el mercado nuevos dispositivos que son portátiles y electroquímicos como NIOX VERO VR ${ }^{\circledR}$. Estos nuevos analizadores se utilizan con frecuencia porque son muy versátiles, pero diversas investigaciones $s^{30-35}$ han reportado valores discrepantes en sus resultados. Por tanto, el dispositivo utilizado debe tenerse en cuenta a la hora de interpretar las concentraciones de $\mathrm{NO}$ exhalado.

\section{Uso clínico}

A continuación, nos referiremos en forma breve al lugar que ocupa la valoración de FeNO en algunas situaciones clínica particulares:

\section{A. Asma}

La inflamación alérgica y eosinofílica son características centrales de la fisiopatología del asma. Estudios han confirmado una fuerte, aunque no siempre absoluta, correlación entre FeNO y eosinofilia en el esputo inducido ${ }^{29,36}$, lavado broncoalveolar ${ }^{37}$ y tejido ${ }^{38}$. Sin embargo, desde el punto de vista clínico el asma se caracteriza por ser heterogéneo en síntomas y tipos de inflamación de vía aérea por lo que se considera un síndrome con fenotipos diferentes. Ha sido demostrado que pacientes con asma severa refractaria a glucocorticoides y eosinofilia persistente en biopsia endobronquial y/o en fluido de lavado broncoalveolar, tienen valores de FeNO significativamente más elevados que pacientes con asma severa refractaria a glucocorticoides y eosinofilia leves, asma moderada y controles sanos ${ }^{39}$. Por lo tanto, la FeNO en pacientes asmáticos puede ser considerada una prueba complementaria para el diagnóstico de asma $^{40}$. Y, por otra parte, en pacientes asmáticos del fenotipo "eosinófilos elevados", FeNO podría tener un impacto clínico relevante tanto en la orientación de la implementación de estrategias terapéuticas como en la identificación pronóstica, dado que se sabe que la reducción de los eosinófilos se asocia a reducción de las exacerbaciones y hospitalizaciones por asma ${ }^{40}$ Por otra parte, debemos aclarar que la FeNO no es un marcador 
de atopia per sé, sino un marcador de inflamación pulmonar eosinofílica inducida por alergenos correlacionando con eosinófilos en el esputo.

Como la FeNO es un biomarcador no invasivo, tiene un excelente potencial para evaluar la gravedad del asma, la capacidad de respuesta al tratamiento con corticosteroides inhalados y la adherencia al tratamiento ${ }^{12}$. En la Tabla 2 entregamos un breve resumen de las recomendaciones actuales de la ATS.

\section{B. Enfermedad pulmonar obstructiva crónica (EPOC)}

La EPOC es definida por la WHO como una enfermedad pulmonar caracterizada por la obstrucción crónica al flujo de aire pulmonar no completamente reversible y de carácter progresivo ${ }^{41}$. Su principal factor de riesgo es la exposición al humo de tabaco y en su fisiopatología, la presencia de hipersecreción, inflamación y elementos de estrés oxidativo en la vía aérea y tejido pulmonar. Desde el punto de vista clínico, la progresión de la EPOC es evidenciada por la progresión del síntoma disnea asociado a manifestaciones sistémicas entre las que se incluyen depresión y trastornos nutricionales, entre otros. Entre las pruebas de soporte diagnóstico para esta entidad, la espirometría tiene un rol central.

Fundado en los resultados de estudios de FeNO en asma, se ha discutido el rol de FeNO en pacientes EPOC. Sin embargo, su evaluación en pacientes EPOC ha mostrado resultados inconsistentes. Un reciente meta-análisis realizado por Lu et al. ${ }^{42}$, analizó 24 estudios y concluyó que

\section{Tabla 2. Recomendaciones de la ATS $^{8}$}

- Recomendamos el uso de FeNO en el diagnóstico de la inflamación de las vías respiratorias eosinofílicas (recomendación fuerte, calidad moderada de la evidencia)

- Recomendamos el uso de FeNO para determinar la probabilidad de respuesta a los esteroides en personas con síntomas respiratorios crónicos, posiblemente debido a una inflamación de las vías respiratorias (recomendación fuerte, evidencia de baja calidad)

- Sugerimos que se pueda usar FeNO para apoyar el diagnóstico de asma en situaciones en las que se necesita evidencia objetiva (recomendación débil, calidad moderada de la evidencia)

- Sugerimos el uso de puntos de corte en lugar de valores de referencia al interpretar los niveles de FeNO (recomendación débil, calidad de evidencia baja)

- Recomendamos tener en cuenta la edad como un factor que afecta a FeNO en niños menores de 12 años (recomendación sólida, evidencia de alta calidad)

- Recomendamos que se use FeNO bajo de menos de 25 ppb (<20 ppb en niños) para indicar que la inflamación eosinofílica y la capacidad de respuesta a los corticosteroides son menos probables (recomendación fuerte, calidad de evidencia moderada)

- Recomendamos que se use FeNO de más de 50 ppb (>35 ppb en niños) para indicar la inflamación eosinofílica y, en pacientes sintomáticos, la capacidad de respuesta a los corticosteroides es probable (recomendación fuerte, calidad moderada de la evidencia)

- Recomendamos que los valores de FeNO entre 25 ppb y 50 ppb (20-35 ppb en niños) se interpreten con cautela y con referencia al contexto clínico. (fuerte recomendación, baja calidad de la evidencia)

- Recomendamos considerar la exposición persistente y / o alta alérgeno como un factor asociado con niveles más altos de FeNO (recomendación fuerte, calidad moderada de la evidencia)

- Recomendamos el uso de FeNO en el monitoreo de la inflamación de las vías respiratorias en pacientes con asma (recomendación fuerte, evidencia de baja calidad)

- Sugerimos utilizar los siguientes valores para determinar un aumento significativo en FeNO: más del $20 \%$ para valores superiores a 50 ppb o más de 10 ppb para valores menores de 50 ppb de una visita a la siguiente (recomendación débil, calidad de evidencia baja)

- Sugerimos utilizar una reducción de al menos 20\% en FeNO para valores superiores a 50 ppb o más de 10 ppb para valores inferiores a 50 ppb como punto de corte para indicar una respuesta significativa a la terapia antiinflamatoria (recomendación débil, calidad baja). de la evidencia) 
los pacientes EPOC presentan valores de FeNO levemente más altos que los controles, y que los pacientes ex fumadores tiene niveles más altos que los fumadores actuales ${ }^{42}$. Interesantemente, el estudio del Erasme University Hospita ${ }^{27}$ publicado a comienzos de 2019, mostró que en pacientes EPOC estables tratados con dosis única de broncodilatador beta-agonista, la valoración de FeNO identificó tres subgrupos de pacientes: i) FeNO no cambia; ii) FeNO aumenta y iii) FeNO disminuye acorde con la extensión del proceso de dilatación. El último grupo representó a 30\% de los pacientes estudiados quienes mostraron una mejoría homogénea de la obstrucción de las vías aéreas hasta la periferia, mientras que en los otros dos grupos se observó una distribución heterogénea de la broncodilatación a predominio de las porciones más proximales de las vías respiratorias proponiendo los autores que la valoración de FeNO antes vs después de una dosis de broncodilatador podría ser de utilidad para identificar pacientes EPOC respondedores a broncodilatación agregando un impacto potencial en resultados clínicos relevantes como las exacerbaciones ${ }^{27}$.

\section{Fibrosis quística}

En la Fibrosis Quística (FQ), la mutación de la proteína transmembrana reguladora de conductancia de la FQ (CFTR) del tejido pulmonar favorece el desarrollo de inflamación en la vía aérea, deshidratación de la capa de líquido de la superficie de la vía aérea, retención de mucus y alto riesgo de infecciones lo que en forma progresiva reduce la función pulmonar ${ }^{43}$.

Los valores de FeNO en pacientes con FQ son muy parecidos a los observados en población sana, esto ha sido interpretado secundario a que la inflamación de las vías aéreas es de predominio neutrofílico $^{44}$. Sin embargo, otros autores han reportado pacientes con FQ y niveles de FeNO más bajos que los controles sanos ${ }^{45,46}$. Se ha hipotetizado que estas diferencias podrías estar fundadas en expresión deficiente de isoforma de NOS, falta de sustratos de NOS, inhibidores endógenos de NOS, difusión deficiente de $\mathrm{NO}$ a través del mucus denso propio de la FQ y descomposición de NO por bacterias que colonizan la vía aérea ${ }^{47}$. Por otra parte, se sabe que bajos niveles de FeNO son más frecuentes en pacientes sin función residual de CFTR por lo que la magnitud del defecto del CFRT es otra variable que considerar ${ }^{47}$. Además, en pacientes con mutaciones del CFRT graves como las de clase III tratados con Ivacaftor, fármaco potenciador del canal CFTR, se ha observado aumento de los valores de FeNO sugiriendo un rol potencial como biomarcador de la restauración de la función del CFTR con este tipo de tratamiento ${ }^{47,48}$.

\section{Disquinesia ciliar primaria}

La disquinesia ciliar primaria (DCP) es una enfermedad congénita de herencia autosómica recesiva que se caracterizada por la movilidad anormal de los cilios presentes en células de tejidos como el respiratorio y gonadal. En el sistema respiratorio, el espectro del defecto ciliar incluye inmovilidad completa, movimiento disquetico e ineficaz y ausencia o aplasia de éstos generando un fenotipo caracterizado por el deterioro en el clearance de vía aérea. A largo plazo, esta condición favorece el desarrollo de infecciones crónicas, inflamación de la vía aérea y el desarrollo de bronquiectasias.

Un estudio de Lundberg et al..$^{49}$ publicado en 1994, mostró valores de NO nasal bajos en pacientes con DCP, lo cual ha sido confirmado por reportes posteriores de otros autores, resultados que han permitido atribuirle a la valoración de $\mathrm{NO}$ un rol en el análisis de screening sensible ante la sospecha de esta patología $a^{50}$. En 2005, ATS y ERS suscribieron las recomendaciones y estandarización para el uso clínica de esta valoración ante la sospecha diagnóstica de DCP. Sin embargo, recientemente el grupo liderado por Collins $\mathrm{SA}^{51}$ ha comunicado que el valor predictivo de esta prueba resultó significativamente buena en pacientes con alta sospecha de DCP, pero con un exceso de falsos positivos en población general.

En relación a controles sanos, pacientes con DCP presentan valores de FeNO bronquial y alveolar bajos ${ }^{52}$. Interesantemente, se ha reportado que pacientes DCP tratados con L-arginina normalizaron los valores de FeNO así como el transporte mucociliar ${ }^{53}$.

\section{Conclusiones}

NO es un biomarcador de inflamación generado principalmente por vía enzimática. En el sistema respiratorio, los cambios de las concentraciones de $\mathrm{NO}$ se asocian a actividad inflamatoria, así como a respuesta a tratamiento.

FeNO es una técnica no invasiva, segura y 
fácil de realizar en pacientes adultos y pediátricos para cuantificar la concentración de $\mathrm{NO}$ en el tracto respiratorio. El carácter no invasivo de la determinación de FeNO permite su utilización repetida sin riesgo adicional para los pacientes. Las aplicaciones clínicas de la valoración de FeNO incluyen diagnóstico, estimador de severidad, respuesta y adherencia al tratamiento y potencialmente, pronóstico ${ }^{54,55}$ permitiendo el desarrollo de una medicina personalizada en pacientes con enfermedades respiratorias como las revisadas en este artículo ${ }^{56}$.

A pesar de que aún se discute acerca de los valores referenciales, se tiene más claridad y consenso para asma: valores de FeNO altos sugieren un control deficiente de la inflamación asociada a la actividad de la enfermedad con presencia de inflamación persistente y necesidad de optimizar el tratamiento antiinflamatorio, mientras que valores de FeNO bajos sugieren niveles bajos de inflamación pudiendo orientar al clínico en el ajuste de terapia farmacológica.

En pacientes EPOC, FeNO parece especialmente útil en pacientes del fenotipo síndrome de sobreposición asma-EPOC (asthma-COPD overlap syndrome, ACOS) quienes desarrollan inflamación con características tanto de asma como de bronquitis crónica/enfisema ${ }^{57,58}$.

Finalmente, es posible proponer que la cuantificación rutinaria de FeNO, reflejo de la inflamación eosinofílica de la vía aérea, podría ayudar a generar algoritmos de tratamiento a la medida de cada paciente permitiendo además guiar los ajustes de acuerdo al perfil de inflamación estimado por FeNO.

\section{Referencias}

1. Palmer R, Ferringe A, Moncada S. Nitric Oxide release accounts for the biological activity of edothelium-derived relaxing factor. Nature. 1987; 327 (524): 6.

2. Alving K. FENO and suspected asthma: better to identify responsiveness to treatment than to label with a diagnosis. Lancet Respir Med. 2018; 6 (1): 3-5.

3. Berry MA, Shaw DE, Green RH, Brightling CE, Wardlaw AJ, Pavord ID. The use of exhaled nitric oxide concentration to identify eosinophilic airway inflammation: An observational study in adults with asthma. Clin Exp Allergy. 2005; 35 (9): 1175-9.

4. Mansur AH, Srivastava S, Sahal A. Disconnect of type
2 biomarkers in severe asthma; dominated by FeNO as a predictor of exacerbations and periostin as predictor of reduced lung function. Respir Med. [Internet] 2018; 143: 31-8. Available from: https://doi.org/10.1016/j. rmed.2018.08.005

5. Smith AD, Cowan JO, Brassett KP, Filsell S, McLachlan C, Monti-Sheehan G, et al. Exhaled nitric oxide: A predictor of steroid response. Am J Respir Crit Care Med. 2005; 172 (4): 453-9.

6. Barnes PJ. Inhaled corticosteroids. Pharmaceuticals 2010; 3 (3): 514-40.

7. Hetherington K, Costello RW, Heaney L. Fractional Exhaled Nitric Oxide (FENO) supression to identify non-adherence in difficult asthma. Thorax. 2016; 71 (Suppl 3): 4-5.

8. Yates DH. Role of exhaled nitric oxide in asthma. Immunol Cell Biol. 2001; 79 (2): 178-90.

9. Korhonen R, Lahti A, Hämäläinen M, Kankaanranta $\mathrm{H}$, Moilanen E. Dexamethasone inhibits inducible nitric-oxide synthase expression and nitric oxide production by destabilizing mRNA in lipopolysaccharide-treated macrophages. Mol Pharmacol. 2002; 62 (3): 698-704.

10. Hattori R, Sase K, Eizawa H, Kosuga K, Aoyama T, Inoue R. Structure and function of nitric oxide synthases. Int J Cardiol. 1994; 47 (Suppl): 71-5.

11. Carroll W, Ruggins N. Managing childhood asthma: Clinical experience with the measurement of fractional exhaled nitric oxide (FeNO). Paediatr Child Heal (United Kingdom) [Internet]. 2014; 24 (6): 260-3. Available from: http://dx.doi.org/10.1016/j.paed.2014.03.007.

12. Ricciardolo FLM, Sorbello V, Bellezza Fontana R, Schiavetti I, Ciprandi G. Exhaled nitric oxide in relation to asthma control: A real-life survey. Allergol Immunopathol (Madr) [Internet]. 2016; 44 (3): 197-205. Available from: http://dx.doi.org/10.1016/j.aller.2015.05.012.

13. Lee M, Rey K, Besler K, Wang C, Choy J. Inmunobiology of Nitric Oxide and Regulation of Inducible Nitric Oxide Synthase. Results Probl Cell Differ [Internet]. 2017; 62: 181-207. Available from: http://link.springer. com/10.1007/978-3-319-54090-0.

14. Goud C, Dipiero A, Lockette WE, Webb RC, Charpie JR. Cyclic GMP-independent mechanisms of nitric oxide-induced vasodilation. Gen Pharmacol. 1999; 32 (1): 51-5.

15. Bonaventura D, de Lima RG, Vercesi JA, da Silva RS, Bendhack LM. Comparison of the mechanisms underlying the relaxation induced by two nitric oxide donors: Sodium nitroprusside and a new ruthenium complex. Vascul Pharmacol. 2007; 46 (3): 215-22.

16. Dweik R, Boggs PB, Erzurum SC, Irvin CG, Leigh MW, 
Lundberg JO. An Official ATS Clinical Practice Guideline: Interpretation of Exhaled Nitric Oxide Leveles (FENO) for Clinical Applications. Am J Respir Crit Care Med. 2011; 184: 602-25.

17. Antosova M, Mokra D, Pepucha L, Plevkova J, Buday T, Sterusky M, et al. Physiology of nitric oxide in the respiratory system. Physiol Res. 2017; 66: S159-72.

18. Pellegrino R, Viegi G, Brusasco V, Crapo RO, Burgos $\mathrm{F}$, Casaburi R, et al. Interpretative strategies for lung function tests. Eur Respir J. 2005; 26 (5): 948-68.

19. Puckett JL, George SC. Partitioned Exhaled Nitric Oxide to Non-Invasively Assess Asthma. Respir Physiol Neurobiol [Internet]. 2008; 30 (163): 1-27. Available from: https://www.ncbi.nlm.nih.gov/pmc/articles/PMC3624763/ pdf/nihms412728.pdf.

20. Lane C, Knight D, Burgess S, Franklin P, Horak F, Legg $\mathrm{J}$, et al. Epithelial inducible nitric oxide synthase activity is the major determinant of nitric oxide concentration in exhaled breath. Thorax. 2004; 59 (9): 757-60.

21. Jiang J, Malavia N, Suresh V, George SC. Nitric oxide gas phase release in human small airway epithelial cells. Respir Res. 2009; 10: 1-8.

22. Measurement O. ATS/ERS recommendations for standardized procedures for the online and offline measurement of exhaled lower respiratory nitric oxide and nasal nitric oxide, 2005. Am J Respir Crit Care Med. 2005; 171 (8): 912-30.

23. Haccuria A, Michils A, Michiels S, Van Muylem A. Exhaled nitric oxide: A biomarker integrating both lung function and airway inflammation changes. J Allergy Clin Immunol. 2014; 134 (3): 554-9.

24. McCormack MC, Aloe C, Curtin-Brosnan J, Diette GB, Breysse PN, Matsui EC. Guideline-recommended fractional exhaled nitric oxide is a poor predictor of health-care use among inner-city children and adolescents receiving usual asthma care. Chest. 2013; 144 (3): 923-9.

25. Jeppegaard M, Veidal S, Sverrild A, Backer V, Porsbjerg C. Validation of ATS clinical practice guideline cut-points for FeNO in asthma. Respir Med [Internet]. 2018; 144: 22-9. Available from: https://doi.org/10.1016/j.rmed.2018.09.014.

26. Essat M, Harnan S, Gomersall T, Tappenden P, Wong R, Pavord I, et al. Fractional exhaled nitric oxide for the management of asthma in adults: A systematic review. Eur Respir J [Internet]. 2016; 47 (3): 751-68. Available from: http://dx.doi.org/10.1183/13993003.01882-2015.

27. Pérez Bogerd S, Michils A, Malinovschi A. COPD patients with peripheral airway obstruction reversibility identified by exhaled nitric oxide. J Breath Res. 2019; 13 (3): 1-46.

28. Hoyte FCL, Gross LM, Katial RK. Exhaled Nitric Oxide: An Update. Immunol Allergy Clin North Am [Inter- net]. 2018; 38 (4): 573-85. Available from: https://doi. org/10.1016/j.iac.2018.06.001

29. Evaristo KB, Saccomani MG, Martins MA, Cukier A, Stelmach R, Rodrigues MR, et al. Comparison between breathing and aerobic exercise on clinical control in patients with moderate-to-severe asthma: Protocol of a randomized trial. BMC Pulm Med. 2014; 14 (1): 1-8.

30. Kim SH, Moon JY, Kwak HJ, Kim S Il, Park DW, Kim JW, et al. Comparison of two exhaled nitric oxide analyzers: The NIOX MINO hand-held electrochemical analyzer and the NOA280i stationary chemiluminescence analyzer. Respirology. 2012; 17 (5): 830-4.

31. Korn S, Telke I, Kornmann O, Buhl R. Measurement of exhaled nitric oxide: Comparison of different analysers. Respirology. 2010; 15 (8): 1203-8.

32. Harnan SE, Tappenden P, Essat M, Gomersall T, Minton J, Wong R, et al. Measurement of exhaled nitric oxide concentration in asthma: A systematic review and economic evaluation of NIOX MINO, NIOX VERO and Nobreath. Health Technol Assess (Rockv). 2015; 19 (82): 1-330.

33. Endo Y, Shirai T, Akamatsu T, Asada K. Comparison of fractional exhaled nitric oxide levels measured using the NIOX VERO and NOA 280i. Ann Allergy, Asthma Immunol. 2017; 119 (4): 383-5.

34. Borrill Z, Clough D, Truman N, Morris J, Langley S, Singh D. A comparison of exhaled nitric oxide measurements performed using three different analysers. Respir Med. 2006; 100 (8): 1392-6.

35. Müller KC, Jörres RA, Magnussen H, Holz O. Comparison of exhaled nitric oxide analysers. Respir Med. 2005; 99 (5): 631-7.

36. Berlyne GS, Parameswaran K, Kamada D, Efthimiadis A, Hargreave FE. A comparison of exhaled nitric oxide and induced sputum as markers of airway inflammation. J Allergy Clin Immunol. 2000; 106 (4): 638-44.

37. Warke TJ, Fitch PS, Brown V, Taylor R, Lyons JDM, Ennis $\mathrm{M}$, et al. Exhaled nitric oxide correlates with airway eosinophils in childhood asthma. Thorax [Internet]. 2002; 57 (5): 383-7. Available from: http://www.pubmedcentral.nih.gov/articlerender.fcgi?artid=1746317\&tool $=$ pmcentrez\&rendertype $=$ abstract.

38. Brightling CE, Symon FA, Birring SS, Bradding P, Wardlaw AJ, Pavord ID. Comparison of airway immunopathology of eosinophilic bronchitis and asthma. Thorax. 2003; 58 (6): 528-32.

39. Silkoff PE, Lent AM, Busacker AA, Katial RK, Balzar $\mathrm{S}$, Strand $\mathrm{M}$, et al. Exhaled nitric oxide identifies the persistent eosinophilic phenotype in severe refractory asthma. J Allergy Clin Immunol. 2005; 116 (6): 1249-55.

40. Green RH, Brightling CE, McKenna S, Hargadon B, 
Parker D, Bradding P, et al. Asthma exacerbations and sputum eosinophil counts: A randomised controlled trial. Lancet. 2002; 360 (9347): 1715-21.

41. WHO | COPD: Definition [Internet]. [cited 2019 Dec 30]. Available from: https://www.who.int/respiratory/ copd/definition/en/

42. Lu Z, Huang W, Wang L, Xu N, Ding Q, Cao C. Exhaled nitric oxide in patients with chronic obstructive pulmonary disease : a systematic review and meta-analysis. Int J Chron Obstruct Pulmon Dis. 2018; 13: 2695-705.

43. Schultz A, Stick S. Early pulmonary inflammation and lung damage in children with cystic fibrosis. Respirology. $2015 ; 20$ (4): 569-78.

44. Armstrong DS, Grimwood K, Carlin JB. Lower Airway Inflammation in Infants and Young Children with Cystic Fibrosis. Am J Respir Crit Care Med [Internet]. 1997; 156 (4): 1197-204. Available from: http://www.ncbi.nlm. nih.gov/pubmed/9351622\%0Ahttp://www.atsjournals. org/doi/abs/10.1164/ajrccm.156.4.96-11058.

45. Hubert D, Aubourg F, Fauroux B, Trinquart L, Sermet I, Lenoir G, et al. Exhaled nitric oxide in cystic fibrosis: Relationships with airway and lung vascular impairments. Eur Respir J. 2009; 34 (1): 117-24.

46. Elphick HE, Demoncheaux EA, Ritson S, Higenbottam TW, Everard ML. Exhaled nitric oxide is reduced in infants with cystic. Thorax. 2001; 56 (2): 151-2.

47. Korten I, Liechti M, Singer F, Hafen G, Rochat I, Anagnostopoulou $\mathrm{P}$, et al. Lower exhaled nitric oxide in infants with Cystic Fibrosis compared to healthy controls. J Cyst Fibros [Internet]. 2018; 17 (1): 105-8. Available from: https://doi.org/10.1016/j.jcf.2017.05.005.

48. Kotha K, Szczesniak RD, Naren AP, Fenchel MC, Duan LL, McPhail GL, et al. Concentration of fractional excretion of nitric oxide (FENO): A potential airway biomarker of restored CFTR function. J Cyst Fibros [Internet]. 2015; 14 (6): 733-40. Available from: http:// dx.doi.org/10.1016/j.jcf.2015.07.002.

49. Lundberg JON, Weitzberg E, Nordvall SL, Kuylenstierna R, Lundberg JM, Alving K. Primarily nasal origin of exhaled nitric oxide and absence in Kartagener's syn- drome. Eur Respir J. 1994; 7: 1501-4.

50. Horvath I, Loukides S, Wodehouse T, Csiszer E, Cole E, Kharitonov S. Comparison of exhaled and nasal nitric oxide and exhaled carbon monoxide levels in bronchiectatic patients with and without primary ciliary dyskinesia. Thorax [Internet]. 2003; 58 (1): 68-72. Available from: http://ovidsp.ovid.com/ovidweb.cgi?T=JS\&PA$\mathrm{GE}=$ reference $\& \mathrm{D}=$ emed $6 \& \mathrm{NEWS}=\mathrm{N} \& \mathrm{AN}=2003050850$

51. Collins S, Behan L, Harris A, Kerry G, Lucas JS. The dangers of widespread nitric oxide screening for primary ciliary dyskinesia Research letter. Thorax. 2016; 71 (6): 560-1.

52. Walker WT, Liew A, Harris A, Cole J, Lucas JS. Upper and lower airway nitric oxide levels in primary ciliary dyskinesia , cystic fibrosis and asthma. Respir Med [Internet]. 2013; 107 (3): 380-6. Available from: http:// dx.doi.org/10.1016/j.rmed.2012.11.021

53. Loukides S, Kharitonov S, Wondehouse T, Cole PJ, Barnes PJ. Effect of arginine on mucociliary function in primary ciliary dyskinesia. Lancet. 1998; 352: 371-2.

54. Heaney LG, Busby J, Bradding P, Chaudhuri R. Remotely Monitored Therapy and Nitric Oxide Suppression Identifies Nonadherence in Severe Asthma. Am J Respir Crit Care Med. 2019; 199 (4): 454-64.

55. Smith AD, Ch B, Cowan JO, Brassett KP, Herbison GP, Sc M, et al. Use of Exhaled Nitric Oxide Measurements to Guide Treatment in Chronic Asthma. N Engl J Med. 2005; 352 (21): 2163-73.

56. Donohue JF, Jain N. Exhaled nitric oxide to predict corticosteroid responsiveness and reduce asthma exacerbation rates. Respir Med [Internet]. 2013; 107 (7): 943-52. Available from: http://dx.doi.org/10.1016/j. rmed.2013.02.018

57. Asthma, COPD, and Asthma-COPD Overlap Syndrome - Global Initiative for Chronic Obstructive Lung Disease - GOLD [Internet]. [cited 2019 Dec 30]. Available from: https://goldcopd.org/asthma-copd-asthma-copd-overlap-syndrome/

58. Postma DS, Rabe KF. The asthma-COPD overlap syndrome. N Engl J Med. 2015; 373 (13): 1241-9. 